\title{
On the use of tester stocks to predict the competitive ability of genotypes
}

\author{
MAURO SANTOS, ${ }^{*}$ KEVIN FOWLER \& LINDA PARTRIDGE \\ Institute of Cell, Animal, and Population Biology, University of Edinburgh, West Mains Road, Edinburgh EH9 3JT, \\ Scotland
}

\begin{abstract}
It has been recently claimed that the outcome of competition between two phenotypically indistinguishable strains cannot be predicted from comparisons of their respective performances against a mutant tester stock. Our aim in the present paper is to disprove this claim and to show the potential pitfalls of deriving conclusions from a statistical analysis of experimental designs commonly employed for the study of competitive interactions in genetically homogeneous and heterogeneous mixtures. Using our own data, we conclude that evaluating the competitive interactions of phenotypically indistinguishable wild-type strains by competing them against mutant marked stocks still remains a valuable method.
\end{abstract}

Keywords: body size, competition, mutant, tester strains.

\section{Introduction}

In a recent report Adell et al. (1990) analysed the competitive abilities of two phenotypically indistinguishable wild-type strains of Drosophila melanogaster by competing them against eight different mutants. They used the substitution experimental design (Mather \& Caligari, 1981) and found that there was inconsistency in the results of duocultures when any of the wild-type strains were compared to the mutants. As a result, the authors claimed that 'there is no way of comparing two strains with identical phenotype by means of using a third, marker strain'.

An attempt by L. Partridge and K. Fowler (unpublished observations) and M. Santos et al. (unpublished observations) to assess the correlated responses that selection on thorax length in Drosophila may have on competitive ability during the pre-adult period used lines of flies artificially selected for large thorax length and control lines and they competed them to various extents against mutant-marked base stocks. This procedure was necessary given that the means to distinguish phenotypically the large and control lines was not available as they had overlapping distributions for thorax length under conditions of larval crowding. If the claims raised by Adell et al. (1990) are correct, then a valid conclusion cannot be obtained from this proce-

*Permanent address: Departamento de Genética y Microbiología, Universidad Autónoma de Barcelona, 08193 Bellaterra (Barcelona), Spain. dure, as there would be no way to extrapolate to the results of direct competition between the large and control lines.

The purpose of this paper is to show that the above conclusion is not valid, and to warn against drawing apparently simple conclusions from a quick statistical analysis of the data obtained using the experimental approach devised by Mather \& Caligari (1981). At least in some cases, the inconsistencies revealed may result from features of the experimental design. A detailed analysis of a subset of our own data is presented to illustrate the point.

\section{Materials and methods}

\section{Strains and fly handling}

The strains of Drosophila melanogaster used in this experiment were as follows.

1 The wild-type Dahomey (D), collected in Dahomey in 1970 and maintained since then mass-mated in population cages in $25^{\circ} \mathrm{C}$. They were subject to $12: 12$ light/dark cycle with uncontrolled humidity by introducing three one-third pint bottles containing 60 $\mathrm{ml}$ of fresh Edinburgh medium (Fowler \& Partridge, 1986) once a week and removing them after 4 weeks.

2 A hybrid strain obtained from four laboratory lines (L1-L4) that were derived from the Dahomey wildtype base stock (Wilkinson et al., 1990; L. Partridge \& $\mathrm{K}$. Fowler, unpublished observations). These lines were 
each selected for more than 200 generations for increasing thorax length by picking the 10 largest pairs of flies per line from the first 25 pairs to eclose from one-third pint bottles with $60 \mathrm{ml}$ of fresh Edinburgh medium. These lines were maintained at $25^{\circ} \mathrm{C}$ and the generation time was about 2 weeks.

3 A hybrid strain obtained from four laboratory lines (C1-C4) that were also derived from the Dahomey wild-type base stock. All the procedures were as in $\mathbf{2}$ except that the 10 pairs of flies needed to start each generation for each line were randomly picked from the first 25 pairs to eclose, so these lines served as the control for the selection programme.

4 A sparkling poliert $\left(s p a^{p o l} ; 4\right)$ eye mutant in an otherwise Dahomey genetic background. This strain had been maintained in the laboratory in population cages as the Dahomey base stock.

5 A scarlet-eyed mutant $(s t ; 3-44.0)$ in an otherwise Dahomey genetic background. This strain had also been maintained in the laboratory in population cages as the Dahomey base stock.

The hybrid strains for the large (L) and control (C) flies were obtained as follows (for illustration, we will refer to the L1-L4 lines, but the same applies to the $\mathrm{C} 1-\mathrm{C} 4$ lines): $\mathrm{L} 1 \times \mathrm{L} 2$ and $\mathrm{L} 3 \times \mathrm{L} 4$ reciprocal crosses were made by allowing 40 virgin females and 40 virgin males ( $3-5$ days old) to mate and to produce offspring for $96 \mathrm{~h}$ in one-third pint bottles with $60 \mathrm{ml}$ of Edinburgh medium, and transferring the flies to new bottles every $48 \mathrm{~h}$. Forty $(20+20)$ females and $40(20+20)$ males from the offspring of different $\mathrm{L} 1 \times \mathrm{L} 2$ crosses were mated, respectively, with $40(20+20)$ males and $40(20+20)$ females from the L $3 \times$ L 4 crosses, using the same procedure. The flies raised from these crosses were mixed in equal proportions and used as the parents of the first instar larvae needed for the experiment. To standardize the conditions of rearing of all strains, the flies from the Dahomey wild-type base stock and from the two mutant marker strains were also raised in one-third pint bottles in the same way as the $\mathrm{L}$ and $\mathrm{C}$ strains. All fly handling was carried out at room temperature using $\mathrm{CO}_{2}$ anaesthesia on adults not less than $3 \mathrm{~h}$ from eclosion.

\section{Experimental design}

The experimental design to test the competitive abilities of the wild-type flies against the mutant stocks consisted of adding different numbers $\left(X_{\mathrm{i}}=30,60,120\right.$ and 210$)$ of first instar larvae $( \pm 2 \mathrm{~h})$ of the same genotype (monocultures) or the mutant genotypes (two series of duocultures) to a reference number $(N=30)$ of large, control and Dahomey larvae [i.e. the addition experiment in Mather \& Caligari (1981)].
First instar larvae from the five strains were collected with a small spatula from spoons containing grape juice medium (made by adding $450 \mathrm{ml}$ of sweetened grape-juice and $60 \mathrm{~g}$ agar to $800 \mathrm{ml}$ of water and allowed to set in the spoons; see Fowler \& Partridge, 1986) on which females had been allowed to lay eggs for $2 \mathrm{~h}$ the day before. The 4,950 larvae used in each replicate (see below) were a random sample of the larvae obtained from 8,500 mating pairs $(1,500$ mating pairs for each of the three wild-type strains and 2,000 for each of the mutant stocks). The mating pairs were kept in groups of 100 in cylindric plastic containers with the spoons containing the grape juice medium at the bottom. These flies were continuously fed by adding live yeast to the surface of the medium. However, no live yeast was added to the surface of the spoons needed for collections of first instar larvae as this could have introduced variations in the amount of yeast available per culture.

All cultures were set up in vials $(75 \mathrm{~mm}$ depth, 25 $\mathrm{mm}$ diameter) containing $3 \mathrm{ml} 2.5$ per cent agar gel as a non-nutritive base and $3 \mathrm{ml}$ of food medium added after the agar cooled. The food medium was made by adding $13.75 \mathrm{~g}$ agar, $187.5 \mathrm{~g}$ sugar, $207.5 \mathrm{~g}$ maize meal, $37.5 \mathrm{~g}$ flaked yeast and $6 \mathrm{~g}$ nipagin to $2.2 \mathrm{l}$ of water. The agar gel base was used to render a total volume of $6 \mathrm{ml}$ food per vial, so the surface/volume ratio was kept as considered normal in this type of vial to avoid excessive drying of the medium. No live yeast was added and the amount of yeast (dead) per vial was about $50 \mathrm{mg}$.

Each replicate of the experiment consisted of 39 vials: $15(3 \times 5)$ vials for the monoculture series (hereafter referred to as $\mathrm{L} /-, \mathrm{C} /-$ and $\mathrm{D} /-), 12(3 \times 4)$ vials for the duocultures with $s p a^{\mathrm{pol}}(\mathrm{L} / \mathrm{spa}, \mathrm{C} / \mathrm{spa}$ and $\mathrm{D} / s p a)$, and $12(3 \times 4)$ vials for the duocultures with $s t$ $(\mathrm{L} / s t, \mathrm{C} / s t$ and $\mathrm{D} / s t)$. The full experiment was replicated eight times on eight consecutive days, so a randomized block design was used. All cultures were kept together on the same incubator shelf at $25^{\circ} \mathrm{C}$ on a $12: 12$ light/dark cycle with uncontrolled humidity.

For 10 days from the time of first adult emergence, all cultures were examined twice daily (08:00-11:00 and 19:00-23:00). Emerged adults were placed in small vials at $-20^{\circ} \mathrm{C}$ for further analysis in the month after emergence, when the numbers of wild-type and mutant flies emerging in each vial were recorded and used to calculate the proportion of wild-type first instar larvae successfully developing into adults $(p)$.

\section{Statistical aspects of the experimental design}

The following is a summary of Mather \& Caligari's (1981) statistical analysis. 
The various regression coefficients relating the expression of the character of interest $(Y)$ to density may be estimated from the linear additive model:

$\mathbf{Y}=\mathbf{X} \boldsymbol{\beta}+\boldsymbol{\varepsilon}$

where $\mathbf{Y}$ is the $n \times 1$ vector of observed values, $\beta$ is the $k \times 1$ vector of parameters to be estimated (the number of independent variables is $k-1$ ), $\mathbf{X}$ is the $n \times k$ matrix of the values for a particular independent variable, and $\varepsilon$ is the $n \times 1$ vector of random errors. If the linear model is correct, the residual mean square provides an unbiased estimate of $\sigma^{2}$, the variance among random errors. However, repeated observations (true replicates) of the values of the dependent variable at a given level of the independent variables should always be included in this controlled experiment to obtain a direct estimate of $\sigma^{2}$ (pure error) which is independent of the choice of model. The estimate of pure error provides a basis to test the adequacy of the model being fitted by means of a conventional analysis of variance. If the two estimates of $\sigma^{2}$ are not statistically different from one another, we can pool them to obtain an estimate based on more degrees of freedom (Sokal \& Rohlf, 1981,pp. 285-286).

One important aspect of Mather \& Caligari's (1981) experimental design is that the independent variables are not orthogonal. Hence, the partial regression coefficient and partial sums of squares obtained for an independent variable are the contribution of that particular variable after taking into account the effects of all other independent variables, and will not be the same if the general model is simplified by dropping an unimportant variable (i.e. a variable whose partial regression coefficient does not differ statistically from 0 ).

Data manipulation and analyses were done on a Vax-8800 VMS at the Centro de Cálculo de la Universidad Autónoma de Barcelona. The statistical programs used were part of the BMDP Statistical Software (1988). Each observed value of $p$ was converted into an angle using the standard $p_{\mathrm{a}}=\sin ^{-1} \sqrt{p}$ angular transformation. In the duoculture series estimation of viabilities of wild-type flies, relative to those of mutants $\left(s p a^{\mathrm{pol}}\right.$ or $s t$ ), were expressed as the cross-product ratio $v_{\mathrm{i}}=a /(b+1) \times X_{\mathrm{i}} / 30$,

where $a$ and $b$ are, respectively, the numbers of wildtype and mutant flies raised, and $X_{\mathrm{i}}$ is the number of mutant first instar larvae added to the standard 30 wild-type larvae. $v_{\mathrm{i}}$ is an unbiased estimator of the relative viability (Haldane, 1956). For statistical analysis, this ratio was expressed in terms of natural logarithms to make sampling distributions more normally distributed (Manly, 1985, pp. 8-11). A randomized block design analysis of variance for the variable $\ln v_{\mathrm{i}}$, with strains ( $\mathrm{L}, \mathrm{C}$ and D), densities $(60,90,150$ and 240 larvae/vial) and mutants $\left(s p a^{\mathrm{pol}}\right.$ and $\left.s t\right)$ as fixed treatments, and replicates as a random factor, was carried out. In one out of 312 vials, no flies of a particular (wild-type) genotype emerged (replicate 2 of the L/st duoculture series at the 240 larvae/vial density). In this case Bartlett's (1947) empirical correction of $0+1 /$ $(4 N)$ where $N$ is the reference number of seeded larvae, was used to replace the missing value for $p$. One degree of freedom was lost for this correction, although the effect is negligible and can be overlooked for practical purposes.

\section{Results and Discussion}

The angular transformed proportion of wild-type first instar larvae giving rise to adults $\left(p_{\mathrm{a}}\right)$ from the monoand duocultures are given in Table 1 . Inspection of the data suggested that the regression of $p_{\mathrm{a}}$ of the three indicator genotypes ( $\mathrm{L}, \mathrm{C}$ and $\mathrm{D})$ on density was linear, although the plot of standardized residuals versus $Y$

Table 1 Values of $p_{\mathrm{a}}$ for wild-type flies and viability $\left(v_{\mathrm{i}}\right)$ of wild-type flies relative to that of mutants. Each figure is the average of eight replicates

\begin{tabular}{|c|c|c|c|c|c|}
\hline \multirow[b]{2}{*}{ Culture } & \multicolumn{5}{|c|}{ Density } \\
\hline & 30 & 60 & 90 & 150 & 240 \\
\hline \multicolumn{6}{|l|}{$\mathrm{L} /-$} \\
\hline $\begin{array}{c}p_{\mathrm{a}} \\
\mathrm{L} / s p a\end{array}$ & 42.82 & 38.05 & 37.35 & 36.05 & 32.78 \\
\hline$p_{\mathrm{a}}$ & & 37.57 & 32.86 & 26.54 & 25.43 \\
\hline$v_{\mathrm{i}}$ & & 0.488 & 0.410 & 0.306 & 0.337 \\
\hline \multicolumn{6}{|l|}{$\mathrm{L} / s t$} \\
\hline $\begin{array}{l}p_{\mathrm{a}} \\
v_{\mathrm{i}}\end{array}$ & & 28.42 & 27.83 & 23.17 & 17.69 \\
\hline$v_{\mathrm{i}}$ & & 0.287 & 0.269 & 0.198 & 0.141 \\
\hline \multicolumn{6}{|l|}{$\mathrm{Cl}-$} \\
\hline $\begin{array}{c}p_{\mathrm{a}} \\
\mathrm{C} / \mathrm{spa}\end{array}$ & 41.62 & 39.65 & 39.34 & 37.73 & 35.24 \\
\hline$p_{\mathrm{a}}$ & & 36.61 & 33.39 & 33.20 & 32.57 \\
\hline$v_{\mathrm{i}}$ & & 0.449 & 0.441 & 0.451 & 0.492 \\
\hline \multicolumn{6}{|l|}{$\mathrm{C} / \mathrm{st}$} \\
\hline$p_{\mathrm{a}}$ & & 32.84 & 28.06 & 30.35 & 28.32 \\
\hline$v_{\mathrm{i}}$ & & 0.343 & 0.285 & 0.332 & 0.301 \\
\hline \multicolumn{6}{|l|}{$\mathrm{D} / \mathrm{-}$} \\
\hline $\begin{array}{c}p_{\mathrm{a}} \\
\mathrm{D} / \text { spa }\end{array}$ & 60.20 & 58.79 & 56.28 & 55.68 & 53.75 \\
\hline $\begin{array}{l}p_{\mathrm{a}} \\
v_{i}\end{array}$ & & 61.13 & 56.66 & 54.35 & 57.60 \\
\hline$v_{i}$ & & 0.999 & 1.072 & 1.074 & 1.164 \\
\hline \multicolumn{6}{|l|}{$\mathrm{D} / s t$} \\
\hline$p_{\mathrm{a}}$ & & 52.92 & 55.02 & 55.61 & 50.34 \\
\hline$v_{1}$ & & 0.709 & 0.798 & 0.843 & 0.786 \\
\hline
\end{tabular}


showed that several of the residuals were suspiciously large, indicating that a parabola might have fitted the data better. A logarithmic transformation of the independent variables $\left[\log \left(X_{\mathrm{i}}+1\right)\right]$ was used and tests of the linear model are shown in Table 2. The biological meaning of Table 2 is that proportional changes in the number of larvae added to the reference number (independent variables) produced linear responses in the proportion of wild-type flies raised from the vials (dependent variable; $p_{\mathrm{a}}$ ). It should be emphasized that transformations of the independent variables affect only the form of the model, whereas transformations to stabilize variances or to satisfy normality must be made on the dependent variable (Rawlings, 1988, ch. 11). Although a linear relationship between character and density is desirable from both the statistical point of view and for the biological interpretation of data, it is not essential for the purposes of the present paper.

The residual variation round the regression lines after fitting the model [MS (deviations)] does not differ statistically from the error variation [MS (error)] in any case (Table 2), and from the $F$-values obtained it seems reasonable to pool both estimates of $\sigma^{2}$. A Bartlett's test of the pooled estimates revealed homoscedasticity $\left\langle\chi_{(2)}^{2}=0.13, P=0.935\right)$ and the overall error variance
( $\hat{\sigma}^{2}=67.14463$ with 300 d. f.), pooled over indicator genotypes, appears to offer a better basis to find the variances, covariances and standard errors of the estimates of the various parameters.

The estimates of the parameters obtained for the three wild-type strains, together with their standard errors and covariance among $\hat{\beta}$ within strains, are shown in Table 3a. It is clear that, at the reference density of 30 larvae per vial, the viability per larva of strains $\mathrm{L}$ and $\mathrm{C}$ does not differ significantly, but both values are significantly lower than that obtained for the Dahomey flies. For the $\mathrm{L}$ and $\mathrm{C}$ genotypes, the effects of intergenotypic competition seem to be greater than those of intragenotypic competition in both series of duocultures, the relationship being $\left|\hat{\beta}_{\mathrm{m}}\right|<\left|\hat{\beta}_{\mathrm{dspa}}\right|<\left|\hat{\beta}_{\mathrm{dst}}\right|$. In contrast, for the $\mathrm{D}$ strain, it seems that competition from $s p a^{\text {pol }}$ larvae is less intense than that from individuals of the primary genotype, whereas competition from st larvae is stronger than from individuals of the same genotype.

In the addition design (Mather \& Caligari, 1981), the slope of the duoculture regression $\left(\beta_{\mathrm{d}}\right)$ measures the effect that the competitor strains have on the competitive success of the indicator or primary genotype. As the number of first instar larvae of the indicator

Table 2 Analyses of variance to test the linear model

\begin{tabular}{|c|c|c|c|c|c|c|c|}
\hline \multirow[b]{2}{*}{ Source } & \multirow[b]{2}{*}{ d.f. } & \multicolumn{2}{|l|}{ L } & \multicolumn{2}{|l|}{$\mathrm{C}$} & \multicolumn{2}{|l|}{$\mathrm{D}$} \\
\hline & & MS & $F$ & MS & $F$ & MS & $F$ \\
\hline Explained & 3 & 1505.73 & $21.52^{*}$ & 545.40 & $7.29^{*}$ & 177.10 & 2.62 \\
\hline Deviations & 9 & 40.25 & 0.58 & 15.84 & 0.21 & 34.59 & 0.51 \\
\hline Error & 91 & 69.96 & & 74.84 & & 67.59 & \\
\hline
\end{tabular}

$* P<0.001$.

Table 3 Estimates of the parameters

\begin{tabular}{|c|c|c|c|c|}
\hline Strain & $\beta_{0}$ & $\beta_{m}$ & $\beta_{\mathrm{dspa}}$ & $\beta_{\mathrm{ds} t}$ \\
\hline \multicolumn{5}{|c|}{ (a) All variables included } \\
\hline L & 45.083617 & -4.734369 & -7.743563 & -10.886226 \\
\hline $\mathrm{C}$ & 41.878176 & -2.106879 & -4.138836 & -6.183209 \\
\hline \multirow[t]{2}{*}{$\mathrm{D}$} & 61.064525 & -2.649593 & -1.966040 & -3.908551 \\
\hline & \pm 2.530898 & \pm 1.483646 & \pm 1.483646 & \pm 1.483646 \\
\hline \multicolumn{3}{|c|}{ Covariance } & 1.052551 & \\
\hline \multicolumn{5}{|c|}{ (b) Best subsets for C and D (see text for details) } \\
\hline \multirow[t]{2}{*}{$\mathrm{C}$} & 38.769063 & - & -2.562151 & -4.606525 \\
\hline & \pm 1.298010 & - & \pm 1.006116 & \pm 1.006116 \\
\hline \multirow[t]{2}{*}{ D } & 57.168698 & - & - & -1.932910 \\
\hline & \pm 0.947995 & - & - & \pm 0.878102 \\
\hline
\end{tabular}


genotypes remains constant throughout the duoculture density series, the amount of intragenotypic competition is also constant, and any difference in the regression slopes among indicator competitors must be due to the intergenotypic competition exerted by the associate genotype. Hence, comparisons among $\hat{\beta}_{\mathrm{dspa}}$ and $\hat{\beta}_{\text {dst }}$ values from the three different wild-type strains will give us evidence about (i) the effect that selection for increasing thorax length has on larval competitive ability ( $\mathrm{L}$ versus $\mathrm{C}$ ), and (ii) the effect that adaptation to differences in culture conditions (e.g. low density and low or nil larval and adult competition in $\mathrm{C} 1-\mathrm{C} 4$ lines in contrast with high densities in population cages) has on competitive ability (C versus D). Analysis of covariance for equality of slopes revealed that the slopes of the variable $p_{\mathrm{a}}$ on the covariate $X_{\text {dspa }}$ were not significantly different among the three indicator genotypes, whereas the regression lines for the covariate $X_{\mathrm{dst}}$ were clearly non-parallel. From this analysis and the results of the two a priori planned comparisons, and also from the fact that $\hat{\beta}_{\mathrm{dspa}}$ does not differ statistically from 0 for the $D$ strain, the following conclusion is reached about the competitive abilities of the three indicator genotypes against the two mutant stocks.

Mutant

$$
\text { Competitive ability }
$$

$\begin{array}{ll}\text { spa } a^{\mathrm{pol}} & \mathrm{D} \geq \mathrm{C}: \mathrm{C}=\mathrm{L} \\ \text { st } & \mathrm{D}=\mathrm{C}>\mathrm{L}\end{array}$

Therefore, it seems that a conclusion (using the 5 per cent standard criterion to reject the null hypothesis) about the effect that selection or culture conditions have on larval competitive ability in Drosophila cannot be reached, because the relationships deduced are dependent on the mutant stock used as a competitor. The above inconsistencies apparently give support to the claims raised by Adell et al. (1990).

However, as $\hat{\beta}_{\mathrm{m}}$ does not statistically differ from 0 for the Control or Dahomey strains, and $\hat{\beta}_{\mathrm{dspa}}$ does not differ significantly from 0 for the Dahomey strain, there is a problem about which variables should be included in the model. The analysis would be statistically straightforward if the independent variables were uncorrelated, because then the contribution of any of them to the variance of $p_{\mathrm{a}}$ would be the same regardless of which other variables were already present, and the results from a single least squares analysis would then suffice. Non-orthogonality is always present in Mather \& Caligari's (1981) experimental design (in the present example the correlation for any pair of independent variables is -0.43 , which results in a positive covariance of 1.05 between $\hat{\beta}$ within each indicator genotype as shown in Table $3 \mathrm{a}$ ). The selection of variables thus needs to be studied carefully. The purpose of the analysis will influence the manner in which the model is constructed; Hocking (1976) provides an excellent review of problems related to the elimination of variables in linear regression models.

In the present type of experiment, the usual objective is not a simple description of the behaviour of the response variable in a particular dataset, but estimation of parameters and understanding of the complex competitive interactions displayed by individuals of like or unlike genotypes. One should always be cautious in dropping variables when estimation of parameters is an objective in the analysis, as the variable dropped may truly affect the dependent variable. Taking the duoculture $\mathrm{D} /$ spa series as an example, it could be said that adding $s p a^{\text {pol }}$ larvae to the reference number $N$ of Dahomey larvae will, sooner or later, significantly reduce the proportion of wild-type flies raised, so the variable $X_{\mathrm{dspa}}$ (i.e. the variable whose partial regression coefficient relates the change in $p_{\text {a }}$ for the Dahomey strain to the increase in density in the duocultures with the $s p a^{\text {pol }}$ mutant) should be retained. However, there are risks in taking this decision. We may have no $a$ priori expectation about the outcome of competition between two genotypes, and what might happen outside the range of the experiment can only be determined by collecting more data.

\section{Simplifying the model}

The present data can be used to illustrate the effect that simplifying the model can have on the estimates of $\beta$, and how this can undermine the previous conclusions. In the present experiment the total number of variables is relatively small, and it is easy to compute all possible subset regressions to find the best model. The methods of forward stepwise selection and backward elimination of variables were both used and they identified the same best subset (Table 3b). Estimates of standard errors in Table $3 \mathrm{~b}$ were based on the residual mean square obtained after dropping 'unimportant' independent variables $[\mathrm{MS}($ residual $)=70.18154$, with 101 d.f. for C; and MS(residual $)=65.44963$, with 102 d.f. for $D]$. The partial regression coefficients obtained for the best subset of predictor variables for the Control and Dahomey strains differ markedly from the 'all variables' model.

From Table 3 , comparisons among $\hat{\beta}_{\mathrm{dsp}: \mathrm{t}}$ and $\hat{\beta}_{\mathrm{dst}}$ values yield the following conclusions about the competitive abilities of $L, C$, and D strains.
Mutant
$\begin{array}{ll}s p a^{\text {pol }} & \mathrm{D}>\mathrm{C}>\mathrm{L} . \\ s t & \mathrm{D}>\mathrm{C}>\mathrm{L} .\end{array}$

Competitive ability

Hence, the inconsistencies found in the earlier analysis disappear, and the conclusion is reached that larval 
mortality increases with body size and with evolution in the selection regime, whichever mutant is used as a tester stock.

\section{Analysis of variance for the variable In $v_{i}$}

As the conclusions about the competitive abilities of the three indicator genotypes using the linear regression approach of Mather \& Caligari (1981) are model dependent, it is desirable to analyse the data from a different perspective. This has been done by means of a randomized block design analysis of variance using the variable $\ln v_{\mathrm{i}}$ (see Materials and methods) because the most obvious way to measure larval viability is to express it in terms of fitness values. Given that $v_{\mathrm{i}}$ can be written as $p X_{\mathrm{i}} /(b+1)$, the variables $p_{\mathrm{a}}$ and $\ln v_{\mathrm{i}}$ are expected to be positively correlated. Pearson correlation coefficients for the 64 duoculture vials within each indicator genotype were 0.929 for $\mathrm{L}, 0.956$ for $\mathrm{C}$, and 0.804 for D.

Table 4 shows the analysis of variance on the basis of 192 observations. The following findings emerge. First, there is a highly significant difference in viabilities among wild-type strains. Second, mutant-marked base stocks exert different competitive pressures against wild-type strains ( $s t$ is a stronger competitor than $\left.s p a^{\text {pol }}\right)$. Third, relative viabilities seem to be frequency- and density-independent for C and D strains (albeit a slight but non-significant increase is observed in the $\mathrm{D} /$ spa and $\mathrm{D} /$ st duoculture series), whereas a decrease in relative viabilities for the $\mathrm{L}$ strain is observed as the frequency and density of $s p a^{\mathrm{pol}}$ and $s t$ mutant larvae increases, which results in a significant

Table 4 Analysis of variance for the variable $\ln v_{\mathrm{i}}$

\begin{tabular}{lrrrl}
\hline Source & d.f. & \multicolumn{1}{l}{ SS } & \multicolumn{1}{l}{ MS } & \multicolumn{1}{l}{$F$} \\
\hline Strains $(\mathrm{S})$ & 2 & 59.0816 & 29.5408 & $57.38^{* * *}$ \\
Mutants (M) & 1 & 10.4171 & 10.4171 & $79.95^{* * *}$ \\
Densities (D) & 3 & 1.0877 & 0.3626 & $5.77^{* *}$ \\
Replicates (R) & 7 & 24.9362 & 3.5623 & \\
$\mathrm{~S} \times \mathrm{M}$ & 2 & 1.0147 & 0.5073 & $5.91^{*}$ \\
$\mathrm{~S} \times \mathrm{D}$ & 6 & 3.5967 & 0.5994 & $7.92^{* * *}$ \\
$\mathrm{~S} \times \mathrm{R}$ & 14 & 7.2077 & 0.5148 & \\
$\mathrm{M} \times \mathrm{D}$ & 3 & 0.7110 & 0.2370 & $3.43^{*}$ \\
$\mathrm{M} \times \mathrm{R}$ & 7 & 0.9123 & 0.1303 & \\
$\mathrm{D} \times \mathrm{R}$ & 21 & 1.3189 & 0.0628 & \\
$\mathrm{~S} \times \mathrm{M} \times \mathrm{D}$ & 6 & 0.7486 & 0.1248 & $1.76 \mathrm{~ns}$ \\
$\mathrm{~S} \times \mathrm{M} \times \mathrm{R}$ & 14 & 1.2007 & 0.0858 & \\
$\mathrm{~S} \times \mathrm{D} \times \mathrm{R}$ & 42 & 3.1776 & 0.0757 & \\
$\mathrm{M} \times \mathrm{D} \times \mathrm{R}$ & 21 & 1.4481 & 0.0690 & \\
$\mathrm{~S} \times \mathrm{M} \times \mathrm{D} \times \mathrm{R}$ & 42 & 2.9796 & 0.0709 & \\
\hline
\end{tabular}

ns $=P>0.05 ; * P<0.05 ;{ }^{* *} P<0.01 ;{ }^{* * *} P<0.001$. strain $\times$ density interaction. Comparisons of $\ln v_{\text {i }}$ means for the three wild-type strains revealed that $\mathrm{D}$ is a stronger competitor than $\mathrm{C}$ and $\mathrm{L}$ in both series of duocultures, whereas $\mathrm{C}$ is stronger than $\mathrm{L}$ at the highest densities only (this is also apparent from Table 1 ). This last result confirms and extends the findings of $\mathrm{L}$. Partridge \& K. Fowler (unpublished observations).

As discussed by Mather (1969, p. 536), competition will, in general, become more intense as the density of a population rises. Hence, 'a reduction in density will weaken the action of competitive selection and so reduce the fitness of the better than average genotypes and raise those of the less well-endowed types'. This is observed in the $\mathrm{L} / \mathrm{spa}$ and $\mathrm{L} /$ st duoculture series, where the fitness of genetically large flies, relative to that of mutants, was frequency- and density-dependent with competitive ability decreasing as mutant frequency and density increased. This decrease is steep against the $s t$ mutant, which was observed to be the strongest competitor (Tables 1 and 3). On the other hand, the fact that $v_{i}$ remains approximately equal to one or even rises with density in the $\mathrm{D} /$ spa duoculture series (Table 1) confirms the small effect, within the limits of the experiment, that the addition of $s p a^{\mathrm{pol}}$ larvae to a vial already containing Dahomey wild-type larvae has on the Dahomey absolute viability.

In summary, the conclusions obtained from the analysis of variance and the simplified regression model are fully consistent. Genetically large flies do suffer a higher pre-adult mortality with increasing density than their corresponding controls, and past experience in the selection regime does decrease larval competitive ability. This is true whatever mutant is used as the relative tester stock.

Turning now to Adell et al's (1990) paper, the intergenotypic effects of the eight mutant strains they used on the two wild-type ones (wild and Oregon-R) were estimated by subtracting the monoculture and duoculture slopes $\left(\hat{\beta}_{\mathrm{m}}-\hat{\beta}_{\mathrm{d}}\right)$ given that, in the substitution experimental design $\hat{\beta}_{\mathrm{d}}$ measures both intra- and intergenotypic competitive effects on the performance of the indicator genotype (Mather \& Caligari, 1983; De Miranda \& Eggleston, 1987). However, the absence of detectable intragenotypic competition $\left(\hat{\beta}_{\mathrm{m}} \approx 0\right)$ was observed in the wild strain, and intragenotypic effects were the same as intergenotypic ones $\left(\hat{\beta}_{\mathrm{d}} \approx 0\right)$ in 19 per cent of the cases. It is not readily obvious from their paper how this would modify the conclusion reached if the 'all variables' model was simplified. In any case, they correctly pointed out that in those situations when the absence of intragenotypic competition is observed it might be better to analyse intergenotypic effects only. This can be done by using the variable $v_{\mathrm{i}}$ instead of the proportion of wild-type flies raised, as $v_{\mathrm{i}}$ illustrates 
clearly the changes in adaptive values of the various genotypes with density and frequency.

\section{Conclusions}

The objective of this analysis was to illustrate the effect that dropping unimportant independent variables in linear regression may have on the conclusions we obtained from Mather \& Caligari's (1981) experimental design. Our aim was not to dismiss their approach to analysing competition phenomena. On the contrary, this is a very useful experimental design and can give, when used appropriately, important insights into the complex interactions displayed by organisms which are in competition with one another (Mather et al., 1982; Mather \& Caligari, 1983; De Miranda \& Eggleston, 1988; Hemmat \& Eggleston, 1990a, b). The method is an application of the powerful least squares estimation procedure to competition experiments but misinterpretation of the results can occur if this statistical tool is applied in an inappropriate way (see e.g. Wetherill, 1986; Rawlings, 1988). Interpretation of the data may be difficult in more complex and less clearcut situations than the one reported in this paper. The duoculture series can be expanded as much as we want, with a corresponding increase in the dimensions of the X matrix (Mather \& Caligari, 1981). To compute all possible subsets to obtain the best one could become a major computing problem because the number of models to be evaluated increases exponentially (see Hocking, 1976 pp. 7-14; and Rawlings, 1988 ch. 7; for a discussion of this point).

The use of mutants as tester stocks has been widely used in fitness testing (e.g. Lewontin, 1955; Latter \& Robertson, 1962; Bhalla \& Sokal, 1964; Wallace, 1981, pp. 62-72; Sharp, 1982, 1984). As far as the results of this paper are concerned, we have shown that evaluating the competitive interactions of phenotypically indistinguishable wild-type strains by competing them against mutant marker stocks remains, Adell et al. (1990) claims notwithstanding, a valuable and powerful method.

\section{Acknowledgements}

We would like to thank Professor Bill Hill for statistical advice. This work was carried out while MS was spending a sabbatical leave at the former Department of Zoology, University of Edinburgh. Financial support from the Dirección General de Investigación Científica y Técnica (Spain) and SERC (U.K.) is gratefully acknowledged.

\section{References}

ADELL, J. C., MOYA, A., MOLINA, V. AND GONZÁlEZ-CANDELAS, F. 1990. On the analysis of viability data: an example with Drosophila. Heredity, 65, 39-46.
BARTLETT, M. 1947. The use of transformations. Biometrics, 3 , 39-53.

BHALLA, S. C. AND SOKAL, R. R. 1964. Competition among genotypes in the housefly at varying densities and proportions (the green strain). Evolution, 18, 312-330.

BMDP STATISTICAL SOFTWARE. 1988. University of California Press, Berkeley, CA.

DE MiRANDA, J. R. AND EGgleston, P. 1987. A comparison of substitution and addition designs for the analysis of competitive interactions in Drosophila melanogaster. Heredity, $\mathbf{5 8 , 2 7 9 - 2 8 8 . ~}$

DE MIRANDA, J. R. AND EGGLESTON, P. 1988. Larval competition in Drosophila melanogaster. II. Comparing biological and competitive parameters. Heredity, 60, 213-219.

FOWLER, K. AND PARTRIDGE, L. 1986. Variation in male fertility explains an apparent effect of genotypic diversity on success in larval competition in Drosophila melanogaster. Heredity, 57, 31-36.

HALDANE, J. B. S. 1956. Estimation of viabilities. J. Genet., 54, 294-296.

HEMMAT, M. AND EGGLESTON, P. 1990a. Analysis of competitive interactions in triocultures of Drosophila melanogaster. Heredity, 64, 215-222.

HEMMAT, M. AND EGGLESTON, P. 1990b. The biometrical genetics of competitive parameters in Drosophila melanogaster. Heredity, 64, 223-231.

HOCKiNG, R. R. 1976. The analysis and selection of variables in linear regression. Biometrics, 32, 1-49.

LATTER, B. D. H. AND ROBERTSON, A. 1962. The effects of inbreeding and artificial selection on reproductive fitness. Genet. Res. Camb., 3, 110-138.

LEWONTIN, R. C. 1955. The effects of population density and composition on viability in Drosophila melanogaster. Evolution, 9, 27-41.

MANLy, B. F. J. 1985. The Statistics of Natural Selection. Chapman and Hall, London.

MATHER, K. 1969. Selection through competition. Heredity, 24, 529-540.

MATHER, K. AND CAligari, P. D. S. 1981. Competitive interactions in Drosophila melanogaster. II. Measurement of competition. Heredity, 46, 239-254.

MATHER, K. AND CALIGARI, P. D. S. 1983. Pressure and response in competitive interactions. Heredity, 51, 435-454.

MATHER, K., HILL, J. AND CALigARI, P. D. S. 1982. Analysis of competitive ability among genotypes of perennial ryegrass. Heredity, 48, 421-434.

Rawlings, J. o. 1988. Applied Regression Analysis: A Research Tool. Wadsworth \& Brooks, Pacific Grove, California.

SHARP, P. M. 1982. Competitive mating in Drosophila melanogaster. Genet. Res. Camb., 40, 201-205.

SHARP, P. M. 1984. The effect of inbreeding on competitive male-mating ability in Drosophila melanogaster. Genetics, 106, 601-612.

SOKAL, R. R. AND ROHLF, F. J. 1981. Biometry, 2nd edn. W. H. Freeman, New York.

wallace, B. 1981. Basic Population Genetics. Columbia University Press, New York.

WETHERILl, G. B. 1986. Regression Analysis with Applications. Chapman and Hall, London.

WILKINSON, G. S., FOWLER, K. AND PARTRIDGE, L. 1990. Resistance of genetic correlation structure to directional selection in Drosophila melanogaster. Evolution, 44, 1990-2003. 